EDITORIAL

\title{
Perfect use versus typical use: translating outcomes in the treatment of diabetic macular oedema
}

(c) The Author(s), under exclusive licence to The Royal College of Ophthalmologists 2021

Eye (2022) 36:1-2; https://doi.org/10.1038/s41433-021-01627-6

Diabetic macular oedema (DMO) is a major cause of visual impairment in working age adults, and is the most prevalent vision-threatening form of diabetic retinopathy (DR) [1]. It is estimated that 5.5 million people in the United Kingdom will have diabetes by 2030, highlighting the significant health and economic burden of this condition [2]. Over the last decade, there has been a paradigm shift in the management of patients with DMO. Intravitreal anti-vascular endothelial growth factor therapy (anti-VEGF) is now considered the mainstay of treatment, with landmark trials establishing its efficacy with mean visual gains of between +9.7 and +13.3 letters at 1 year [3-6].

Currently there are two main anti-VEGF therapy in use for DMO in the UK: ranibizumab, which was approved by the National Institute of Health and Care Excellence (NICE) in 2013 [7], and aflibercept, which was approved in 2015 [8]. A third VEGF inhibitor, bevacizumab, is routinely used off-label around the world. Based on the registration trials, the NICE guidelines recommend ranibizumab $0.5 \mathrm{mg}$ to be used monthly for 3 months followed by a pro-re-nata (PRN) approach. Aflibercept $2 \mathrm{mg}$ is recommended for use monthly for 5 months followed by bimonthly injections for the first 12 months. After month 12, treatment interval can be extended based on functional or anatomic parameters $[7,8]$.

Although randomised clinical trials are an essential benchmark by which to judge treatment efficacy on, studies examining real world outcomes also provide valuable information on a more practical level. Indeed, in a recent large scale retrospective study of 28,658 eyes with treatment naïve $\mathrm{DMO}$, electronic medical records revealed patients received a mean of 6.4 intravitreal injections and demonstrated a mean visual gain of only +4.2 letters at 1 year [9]. This is consistent with other observational studies which have shown that patients with DMO receive fewer injections and have lower visual gains compared to those in clinical trials $[10,11]$.

In this edition, Sivaprasad et al. report the UK experience with aflibercept for DMO, providing an insight into local treatment patterns and outcomes in routine clinical practice [12]. The DRAKO study is a prospective multi-centre observational study across the UK of patients with centre-involving DMO treated with aflibercept as part of standard of care. This is a planned 24-month study, including both anti-VEGF treatment naïve patients and those previously treated with anti-VEGF. The interim 12 months results for the 507 patients in the treatment naïve cohort is reported here.

After accounting for drop out, findings for 488 patients (the full analysis set) were reported but only 388 of these had 12 months follow up data available (the per protocol window set). Surprisingly, the results demonstrated that patients in the DRAKO study had a mean visual gain of only +2.5 letters at month 12 . This is significantly lower than the +10.7 letter gain for aflibercept seen in the VIVID ${ }^{\mathrm{DMO}}$ and VISTA ${ }^{\mathrm{DMO}}$ clinical trials and even in the similarly real-world APOLLON observational study conducted in France $[5,13]$. One possible cause for this observation is the higher baseline visual acuity for this cohort of 71.4 letters compared to the initial trials (59.8 and 59.1 letters for VIVID ${ }^{\mathrm{DMO}}$ and VISTA ${ }^{\mathrm{DMO}}$ ), which represents a 'ceiling effect' for potential visual gain [5]. This is reflected in the sub-group with lower baseline visual acuity of $\leq$ 69 letters, where visual gains were more comparable at +7.3 letters at 12 months.

Perhaps of more interest is the lower injection frequency and non-adherence to treatment protocol in the study. At baseline, the intended injection regimen was recorded at each site according to the local standard of care, with 11 different protocols in place across the 35 sites. Most of the recruited patients were treated in sites that intended to follow the NICE guidelines (the summary of product characteristics [SmPC]) of at least 5 loading doses $(n=$ 334 patients, $86.1 \%$ ). However, in practice, only $30.2 \%$ of patients received their intended five loading dose, and even fewer (3.4\%) followed the full SmPC treatment regimen through year one. Given the association between greater injection frequency and better visual outcomes [9], the results in the DRAKO trial may not be that surprising.

However, it begs the question, why do real world studies consistently demonstrate fewer injections and lower adherence to prescribed treatment protocols? This occurs even in a prospective setting such as the DRAKO study where investigators and study participants are aware their involvement is being assessed. There are several potential reasons for this discrepancy. Firstly, participants in randomised clinical trials are highly selective and do not reflect patients in routine clinical practice. In the real world, patients with diabetes are generally sicker with more comorbidities and poorer glycaemic control that would otherwise exclude them from clinical trials. Language, poorer health literacy and differing socioeconomic or cultural background are additional barriers [14]. Secondly, priorities for treatment may vary. Diabetic macular oedema affects a significant proportion who are in the working age group, where time off work to attend appointments may act as a disincentive to treatment. The visual significance of their DMO may not be as meaningful to some patients despite a more objective change in vision. Thirdly, access to treatment may be a limitation with clinic capacity limiting the ability for more intensive treatment. Physician preference may also influence this, with perhaps the perception that DMO is less time-critical and can be initially observed.

Of course, these issues of adherence and injection frequency are not unique to DMO. Similar findings are seen for other intravitreal injection indications such as age-related macular degeneration and retinal vein occlusion [15]. So how do we address this potential conflict? Perhaps one way is to develop better methods of assessing adherence and the true versus potential delivery of any treatment. If we look to the contraceptive 
literature, it has long been recognised the effectiveness of any intervention in prevention of pregnancy is based on two metrics: 'perfect use' and 'typical use' [16]. By recognising real-world usage and its limitations, we can begin to develop better strategies to counteract these barriers and look to optimise treatment outcomes for our patients in a meaningful way.

\section{Mali Okada ${ }^{1 凶}$ \\ ${ }^{1}$ Royal Victorian Eye and Ear Hospital, Melbourne, Australia. 凶email: Mali.Okada@eyeandear.org.au}

\section{REFERENCES}

1. Tan GS, Cheung N, Simó R, Cheung GCM, Wong TY. Diabetic macular oedema. Lancet Diabetes Endocrinol. 2017;5:143-55.

2. Whicher CA, O'Neill S, Holt RIG. Diabetes in the UK: 2019. Diabet Med. 2020;37:242-7.

3. Elman MJ, Aiello LP, Beck RW, Bressler NM, Bressler SB, Edwards AR, et al. Randomized trial evaluating ranibizumab plus prompt or deferred laser or triamcinolone plus prompt laser for diabetic macular edema. Ophthalmology. 2010;117:1064-1077.e35.

4. Brown DM, Nguyen QD, Marcus DM, Boyer DS, Patel S, Feiner L, et al. Long-term outcomes of ranibizumab therapy for diabetic macular edema: the 36-month results from two phase III trials: RISE and RIDE. Ophthalmology. 2013;120: 2013-22.

5. Heier JS, Korobelnik J-F, Brown DM, Schmidt-Erfurth U, Do DV, Midena E, et al. Intravitreal aflibercept for diabetic macular edema: 148-week results from the VISTA and VIVID studies. Ophthalmology. 2016;123:2376-85.

6. Wells JA, Glassman AR, Ayala AR, Jampol LM, Bressler NM, Bressler SB, et al. Aflibercept, bevacizumab, or ranibizumab for diabetic macular edema: two-year results from a comparative effectiveness randomized clinical trial. Ophthalmology. 2016;123:1351-9.

7. Anon. National Institute of Health and Care Excellence Technology appraisal guidance (TA274): Ranibizumab for treating diabetic macula oedema. 2013.

8. Anon. National Institute of Health and Care Excellence Technology appraisal guidance (TA346): Aflibercept for treating diabetic macula oedema. 2015.

9. Ciulla TA, Bracha P, Pollack J, Williams DF. Real-world outcomes of anti-vascular endothelial growth factor therapy in diabetic macular edema in the United States. Ophthalmol Retin. 2018;2:1179-87.
10. Blinder KJ, Dugel PU, Chen S, Jumper JM, Walt JG, Hollander DA, et al. Anti-VEGF treatment of diabetic macular edema in clinical practice: effectiveness and patterns of use (ECHO Study Report 1). Clin Ophthalmol. 2017;11:393-401.

11. Cantrell RA, Lum F, Chia Y, Morse LS, Rich WL III, Salman CA, et al. Treatment patterns for diabetic macular edema: an intelligent research in sight (IRIS ${ }^{\circledR}$ ) registry analysis. Ophthalmology 2020;127:427-9.

12. Sivaprasad S, Ghanchi F, Kelly SP, Kotagiri A, Talks SJ, Scanlon P, et al. Evaluation of standard of care intravitreal aflibercept treatment of diabetic macular oedema treatment-naïve patients in the UK: DRAKO study 12-month outcomes. Eye (Lond). 2021.

13. Korobelnik J-F, Daien V, Faure C, Tadayoni R, Giocanti-Auregan A, Dot C, et al. Real-world outcomes following 12 months of intravitreal aflibercept monotherapy in patients with diabetic macular edema in France: results from the APOLLON study. Graefes Arch Clin Exp Ophthalmol. 2020;258:521-8.

14. Rose MA, Vukicevic M, Koklanis K. Adherence of patients with diabetic macular oedema to intravitreal injections: A systematic review. Clin Exp Ophthalmol. 2020;48:1286-98.

15. Ehlken C, Ziemssen F, Eter N, Lanzl I, Kaymak H, Lommatzsch A, et al. Systematic review: non-adherence and non-persistence in intravitreal treatment. Graefe's Arch Clin Exp Ophthalmol. 2020;258:2077-90.

16. Trussell J. Understanding contraceptive failure. Best Pr Res Clin Obstet Gynaecol. 2009;23:199-209.

\section{AUTHOR CONTRIBUTIONS}

M.O is responsible for the manuscript.Compliance with ethical standards

\section{COMPETING INTERESTS}

M.O receives honorarium from Bayer and Allergan.

\section{ADDITIONAL INFORMATION}

Correspondence and requests for materials should be addressed to M.O.

Reprints and permission information is available at http://www.nature.com/ reprints

Publisher's note Springer Nature remains neutral with regard to jurisdictional claims in published maps and institutional affiliations. 